\title{
Ultraviolet-Visible Plasmonic Properties of Gallium Nanoparticles Investigated by Variable-Angle Spectroscopic and Mueller Matrix Ellipsometry
}

Yang Yang, ${ }^{\dagger}$ Neset Akozbek, ${ }^{\ddagger}$ Tong-Ho Kim, ${ }^{\S}$ Juan Marcos Sanz, $"$ Fernando Moreno," Maria Losurdo, ${ }^{\perp}$ April S. Brown, ${ }^{\S}$ and Henry O. Everitt* ${ }^{* \dagger, \#}$

${ }^{\dagger}$ Department of Physics, Duke University, Durham, North Carolina 27708, United States

${ }^{\ddagger}$ AEgis Technologies Group Inc., 410 Jan Davis Drive, Huntsville, Alabama 35806, United States

${ }^{\S}$ Department of Electrical and Computer Engineering, Duke University, Durham, North Carolina 27708, United States

"Grupo de Óptica, Departamento Física Aplicada, Universidad de Cantabria, 39005 Santander, Spain

${ }^{\perp}$ Institute of Inorganic Methodologies and of Plasmas-CNR and INSTM, Via Orabona, 4-70126 Bari, Italy

\#C.M. Bowden Laboratory, Army Aviation \& Missile RD\&E Center, Redstone Arsenal, Alabama 35898, United States

ABSTRACT: Self-assembled, irregular ensembles of hemispherical Ga nanoparticles (NPs) were deposited on sapphire by molecular beam epitaxy. These samples, whose constituent unimodal or bimodal distribution of NP sizes was controlled by deposition time, exhibited localized surface plasmon resonances tunable from the ultraviolet to the visible (UV/ vis) spectral range. The optical response of each sample was characterized using a variable-angle spectroscopic ellipsometer, and the dielectric response of the ensemble of NPs on each sample was parametrized using Lorentz oscillators. From this,

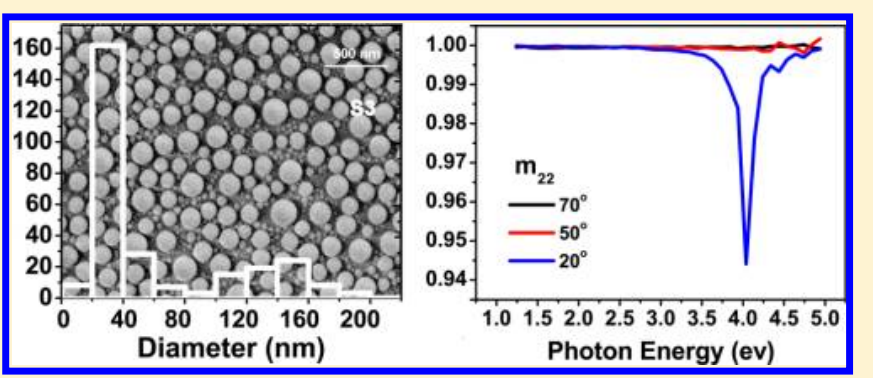
a relationship was found between NP size and the deduced Lorentzian parameters (resonant frequency, damping, oscillator strength) for most unimodal and bimodal samples at most frequencies and angles of incidence. However, for samples with a bimodal size distribution, Mueller matrix ellipsometry revealed nonspecular scattering at particular frequencies and angles, suggesting a resonant interparticle coupling effect consistent with recently observed strong local field enhancements in the ultraviolet.

KEYWORDS: plasmonics, ultraviolet, gallium, nanoparticles, variable-angle spectroscopic ellipsometry, variable-angle Mueller matrix ellipsometry

$\mathrm{R}$ esearch into the optical response of confined electronic plasma oscillations in metallic nanostructures has become the foundation of modern plasmonics. Metallic nanostructures are routinely fabricated with desired spatial geometries for specific applications including single-molecule detection, enhanced fluorescence, toxic remediation, and catalysis. ${ }^{1-6}$ Because the optical response depends sensitively on nanostructure composition, size, and shape, increasingly sophisticated characterization tools are required: aggregate behavior may be characterized by absorption spectroscopy or dark-field microscopy, while single-particle behavior may be characterized by confocal microscopy, near-field scanning optical microscopy, and cathodoluminescence. $^{7-12}$ These tools provide incredibly detailed understanding of the absorption and scattering properties of plasmonic metal nanoparticles, especially when complemented by sophisticated electromagnetic modeling techniques such as finite difference time domain, finite element codes, and the discrete dipole approximation. ${ }^{13-19}$

Of growing interest is the need to monitor the plasmonic properties of substrate-supported nanoparticle (NP) ensembles during fabrication. Because it is impractical to use single-particle imaging tools for this application, advances in the more traditional tools are needed to understand single-particle behaviors from aggregate measurements. Spectroscopic ellipsometry (SE) has been used to monitor how NP ensembles alter the polarization state of an incident light beam as a function of wavelength and incidence angle. ${ }^{20-24}$ Recently, a fixed incidence angle spectroscopic ellipsometer mounted on a molecular beam epitaxy (MBE) chamber has been used to monitor in real time the deposition of nanometer-scale metallic films and NPs through the dynamical evolution of the associated plasmon resonance. $^{25-30}$ Specifically, this technique has been used to demonstrate the correlation between mean NP size and the inplane and out-of-plane localized surface plasmon resonance (LSPR) modes of substrate-supported Ga NPs. ${ }^{20,29,30}$ This approach allows the operator to tune the plasmon resonance to a desired wavelength on any substrate, providing real-time

Received: January 31, 2014 


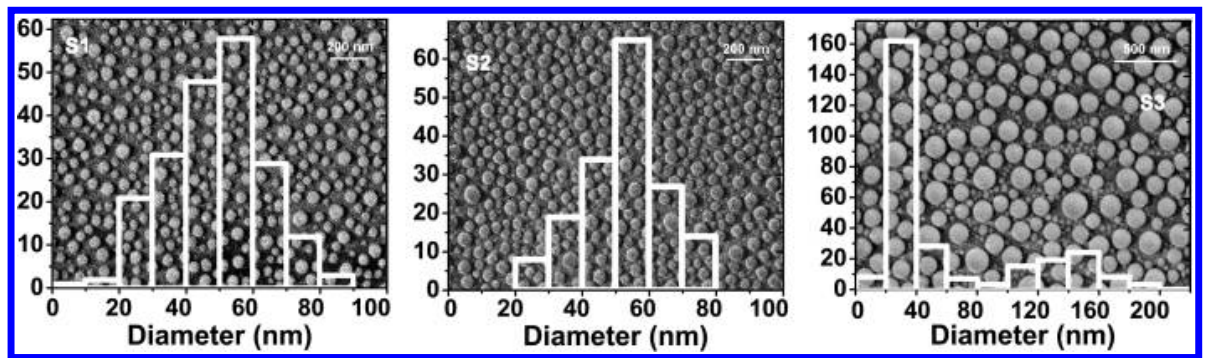

Figure 1. (a) SEM imagery of three ensembles of hemispherical Ga NPs deposited on sapphire, overlaid by a histogram measuring the distribution of NP diameters.

feedback for fabricating structures optimized for plasmonic applications such as UV-SERS, ${ }^{31,32}$ but a deeper analysis is needed to reveal how the measured plasmon resonance frequencies and widths of self-assembled NP ensembles relate to the mean NP size and spatial distribution, respectively. This problem is exacerbated by the fact that LSPRs depend also on the substrate and inter-NP interactions.

Advancing from fixed-angle to variable-angle SE (VASE) makes it possible to analyze much more complex structures. ${ }^{33,34}$ A variable-angle spectroscopic ellipsometer can measure both the angle-dependent response and the Mueller matrix components needed to provide a complete polarimetric analysis. ${ }^{35,36}$ From these measurements, the full optical response of a complex, selfassembled NP ensemble may be described by an effective dielectric function that depends on particle size, shape, and distribution. The conversion of measured VASE data to dielectric functions that accurately describe how the samples scatter incident illumination depends sensitively on the model used, but tremendous insight may be gleaned from reasonably simple, physically meaningful approximations. ${ }^{21,22,24,37}$

Here we report a series of VASE and variable-angle Mueller matrix (VAMM) measurements on self-assembled irregular ensembles of truncated spherical gallium NPs grown by MBE on sapphire substrates whose size distribution was spatially characterized by scanning electron microscopy (SEM). We show that for the majority of samples fabricated a Lorentz oscillator model with a damping term inversely proportional to the NP diameter accurately reproduces the salient behaviors. This model also accurately predicts the wavelength- and angledependence of the critical terms in the measured Mueller matrix characterizing the polarimetric scattering by these samples. Together, these findings indicate that the plasmonic behavior of self-assembled gallium NP ensembles smaller than $100 \mathrm{~nm}$ in diameter and with a unimodal size distribution is dominated by the isolated, single-particle response of the NPs closest to the average diameter.

However, for samples exhibiting a bimodal distribution of larger NPs surrounded by a halo of smaller NPs, deviations from the Lorentzian behavior are observed in ways that depend sensitively on incidence angle and wavelength. Nonspecular scattering and depolarizing features in the Mueller matrix suggest the coupling of NPs alters the plasmonic performance in a manner that may explain the strong local field enhancement seen in a recent demonstration of ultraviolet surface-enhanced Raman spectra. ${ }^{31}$ This insight suggests that VAMM analyses such as presented here may be used as a practical tool to characterize heterogeneous arrays of metal NPs to ascertain the spectral regions in which the strongest local field enhancements occur.

\section{SYNTHESIS AND SPATIAL AND SPECTRAL CHARACTERIZATION OF GA NP ENSEMBLES}

Gallium (Ga) NPs were fabricated for this study for two technologically important reasons. First, unlike gold and silver, gallium has plasmonic activity in the ultraviolet: the plasma energy and damping constant are 14.0 and $1.54 \mathrm{eV}$, respectively. ${ }^{13,31,32,38}$ Because of growing interest in UV plasmonics, investigations of Ga NPs are particularly timely. ${ }^{31,39-41}$ Second, during room-temperature deposition gallium forms hemispherical droplets. This geometry simplifies analysis of the relationship between NP size and the measured LSPR modes, especially in the UV, where there is no interference from interband absorption. This hemispherical form is preserved upon exposure to air by a monolayer-thick, self-forming and selfterminating native oxide. ${ }^{29,42}$

Ga NPs were deposited onto inert, single-side polished sapphire substrates held at room temperature using a Veeco GEN II plasma-assisted MBE system. ${ }^{43}$ Under ultra-highvacuum conditions, the NPs self-assembled during deposition with a constant $\mathrm{Ga}$ beam equivalent pressure of $2.0 \times 10^{-7}$ Torr, corresponding to $19 \mathrm{~nm} / \mathrm{min}$ of $\mathrm{Ga}$ in the thin-film approximation. By keeping a constant beam equivalent pressure and growth rate, the amount of Ga deposited on the substrate was controlled by deposition "dosage" time. Assuming a gallium fcc lattice constant of $a=0.45 \mathrm{~nm}=$ one monolayer (ML), the deposition rate was $42 \mathrm{ML} / \mathrm{min}$.

A variety of samples were prepared, each under identical conditions except that Ga dosage was increased successively from one sample to the next. Previous work on liquid Ga nanodroplets has shown that by undercooling and superheating they can maintain a liquid phase well below room temperature. ${ }^{43}$ Transmission electron microscopy revealed that our NPs are in a homogeneously amorphous liquid state surrounded by a stable, thin $(<1 \mathrm{~nm})$ layer of native oxide. Scanning electron microscopy reveals that NPs grow monotonically larger with increasing dosage (Figure 1). Specifically, samples S1 and S2 show clear unimodal size distributions (mean diameter $\sim 50 \mathrm{~nm}$ ), S1 being more polydisperse than S2 (distribution widths of 35 and $23 \mathrm{~nm}$, respectively). However, the size distribution becomes bimodal as the dosage increases, and the mean diameters of the S3 NPs are 32 and $140 \mathrm{~nm}$, respectively. The smaller NPs in S3 fill the interstices between the larger NPs, likely coalescence residue of the NPs from which they formed. ${ }^{44}$

\section{SPECTROSCOPIC ELLIPSOMETRY OF GA NPS}

During deposition, an in situ UVISEL Jobin-Yvon spectroscopic ellipsometer spanning the $1.5-6.0 \mathrm{eV}$ region, with an incident angle $\theta_{i}=70^{\circ}$ from normal, monitored the collective optical response of the Ga NP ensemble on the sapphire substrate. ${ }^{29,30}$ In an SE experiment, linearly polarized light illuminates the 

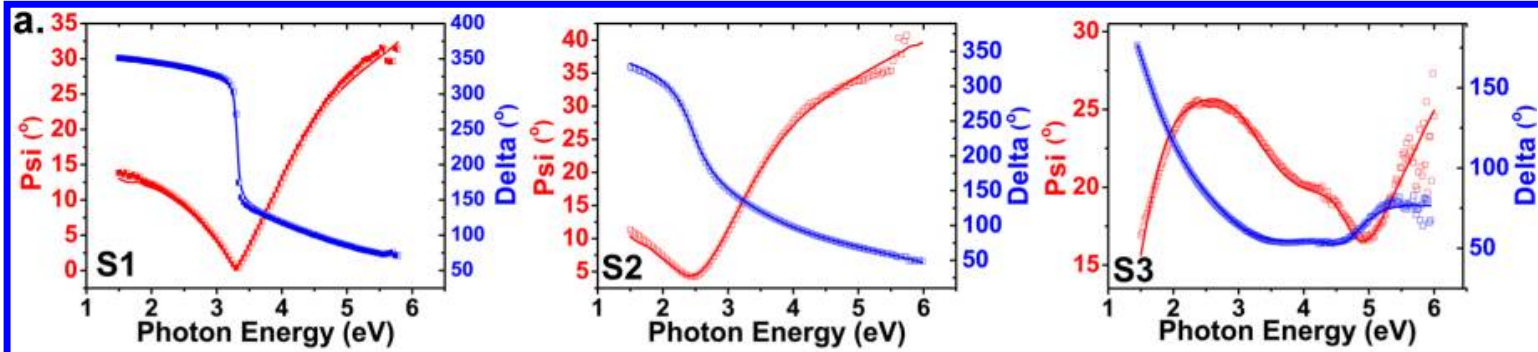

b.
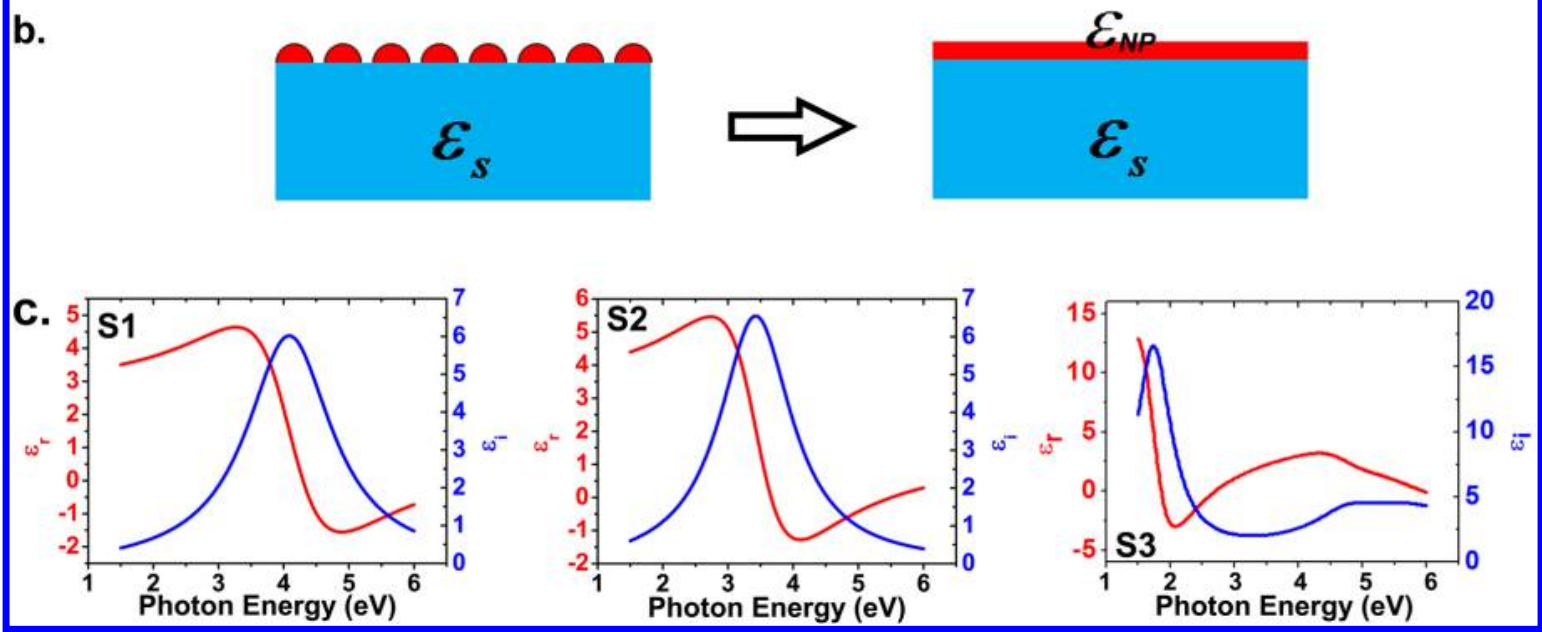

Figure 2. (a) Measured $\Psi$ and $\Delta$ data from the fixed $\theta_{i}=70^{\circ}$ spectroscopic ellipsometer mounted on the MBE chamber for each of the three samples shown in Figure 1. The data are overlaid by a fit using the Lorentz oscillator model, from which the resonance frequency $\omega_{0}$, damping parameter $\gamma$, fractional strength $f$, and effective thickness $\delta$ are obtained. (b) Diagrams illustrating the approximations used to model the NP ensembles. (c) Real and imaginary portions of the dielectric function deduced from the parameters obtained in the fit in (a).

interrogated sample, and the complex ratio $\rho=r_{\mathrm{p}} / r_{\mathrm{s}}$ of reflected p- and s-polarized light is measured as a function of photon energy. We will call in-plane/out-of-plane excitation that which is produced by the component(s) of the incident electric field parallel/perpendicular to the substrate. The sample-induced relative changes in amplitude and phase of the polarizations are given by

$$
\rho=\tan \Psi e^{i \Delta}
$$

where the angles $\Psi$ and $\Delta$ correspond to the amplitude ratio and phase difference of the reflection coefficients, respectively. Examples of measured $\Psi$ and $\Delta$ for the three Ga NP samples in Figure 1 are presented in Figure 2a. For a semi-infinite substrate with an overlayer of Ga NPs in a semi-infinite air medium, the real and imaginary parts of the pseudodielectric function can be derived from $\Psi$ and $\Delta$ through $^{45}$

$$
\begin{aligned}
\langle\varepsilon(\omega)\rangle & =\left\langle\varepsilon_{\mathrm{r}}(\omega)\right\rangle+\left\langle\varepsilon_{\mathrm{i}}(\omega)\right\rangle \\
& =\sin ^{2} \theta_{\mathrm{i}}\left[1+\left(\frac{1-\rho}{1+\rho}\right)^{2} \tan ^{2} \theta_{\mathrm{i}}\right]
\end{aligned}
$$

The pseudodielectric function extracted from eq 2 provides quite useful in situ diagnostics if monitored during deposition. $^{20,24-26,29,30,46}$ The samples were subsequently measured more extensively ex situ with a J. A. Woollam Co., Inc. VASE system to collect angle- and energy-resolved VAMM and scattering spectra.

To analyze the optical response of a Ga NP ensemble on sapphire, we begin with a microscopic description by assuming the electrons oscillate with a Lorentzian behavior in response to the applied field at frequency $\omega .{ }^{20}$ Assuming for the moment that the Ga NP ensemble macroscopically behaves as an isotropic layer of effective thickness $\delta$ (Figure 2b), its dielectric response can be parametrized by

$$
\varepsilon(\omega)=\varepsilon_{\infty}+\sum_{j=1}^{N} \frac{f_{j} \omega_{0 j}^{2}}{\omega_{0 j}^{2}-\omega^{2}+i \gamma_{j} \omega}
$$

where $N$ is the number of oscillators, each of which is characterized by a resonant frequency $\omega_{0}$, damping parameter $\gamma$, and fractional strength $f$. These parameters and $\varepsilon_{\infty}$ are then adjusted by nonlinear least-squares analysis to fit the measured $\Psi, \Delta$ data and obtain the parameters presented in Table 1 .

Table 1. Fitted Parameters from $70^{\circ}$ SE Data for Three Samples Using Lorentzian Model, Compared to the SEMMeasured Mean NP Diameter(s) for Each Sample

$\begin{array}{cccccc} & d / \mathrm{nm} & \varepsilon_{\infty} & f_{j} & \omega_{0 j} / \mathrm{eV} & \gamma_{j} / \mathrm{eV} \\ \text { S1 } & 48 & 0.95 \pm 0.05 & 3.23 \pm 0.04 & 4.16 \pm 0.02 & 1.59 \pm 0.02 \\ \text { S2 } & 52 & 1.44 \pm 0.02 & 3.95 \pm 0.06 & 3.49 \pm 0.02 & 1.35 \pm 0.02 \\ \text { S3 } & 32, & 0.95 \pm 0.09 & 6.24 \pm 0.24 & 1.77 \pm 0.01 & 0.69 \pm 0.02 \\ & 140 & & 2.25 \pm 0.10 & 5.95 \pm 0.10 & 3.27 \pm 0.13 \\ & & & 0.26 \pm 0.08 & 4.75 \pm 0.04 & 1.02 \pm 0.18\end{array}$

Indeed, the validity of the isotropic model has also been checked by acquiring ellipsometric spectra for unimodal samples at various angles of incidence and rotating the sample in the plane. No significant variation was observed. This isotropic response derives from the random arrangement of the amorphous liquid phase NPs. Here, a three-phase model describes the incident 
medium (air), thin Ga NP ensemble layer, and semi-infinite (noback reflected light) $\mathrm{Al}_{2} \mathrm{O}_{3}$ substrate (Figure $2 \mathrm{~b}$ ). The fits, overlaid in Figure 2(a), quite accurately reproduce the measured $\theta_{\mathrm{i}}=70^{\circ}$ data below $5 \mathrm{eV}$ where the data are less noisy. The corresponding estimates of the dielectric functions retrieved for these three samples are shown in Figure 2c.

Several features are worth noting here. In the spectral range analyzed, the unimodal samples $S 1$ and $S 2$ could be characterized by a single Lorentzian describing the dominant lower energy inplane LSPR mode caused by electron plasma oscillating parallel to the substrate surface and excited by both the s-polarized light and the in-plane component of the p-polarized light. The out-ofplane LSPR mode, produced by the component of the incident electric field perpendicular to the substrate, occurs at energies above the range of the spectrometer. Indeed, Mie calculations predict that for hemispherical NPs with an effective diameter of $50 \mathrm{~nm}$, the out-of-plane dipolar LSPR mode should occur above $7 \mathrm{eV}$. By contrast, the bimodal sample S3 required three Lorentzian oscillators respectively representing the in-plane and out-of-plane LSPR modes at $\omega_{0}=1.77$ and $4.75 \mathrm{eV}$ for the larger NPs and the in-plane mode $\left(\omega_{0}=5.95 \mathrm{eV}\right)$ for the smaller NPs. These assignments are also supported by Mie theory calculations. ${ }^{47}$ All three samples yield a value for $\varepsilon_{\infty} \approx 1$.

But how may this analysis, based the assumption of an isotropic Ga film on sapphire, be understood in the context of the actual self-assembled Ga NP ensembles? To what extent can we correlate the parameters in eq 3 with the characteristics observed in the SEM image: the size and shape of the Ga NPs, their polydispersity, their mutual interaction, the interaction with the substrate, and multiple scattering due to the particle surface density? Consider how the fitted resonance frequencies $\omega_{0}$, damping parameters $\gamma$, and oscillator strengths $f$ vary with the mean cross sectional diameter of the NPs $d$. Inspection of Table 1 reveals that for the lowest frequency mode (i.e., in-plane dipole), both $\omega_{0}(d)$ and $\gamma(d)$ decrease while $f(d)$ increases with increasing $d$. Since the Lorentz model reflects the damped oscillatory motion of free electrons, the dependence of $\omega_{0}$ and $f$ on $d$ does not arise from any model-dependent assumptions about confinement. Instead, this dependence reflects the relationship between the mean size of the NPs and the frequency and strength of the induced dipolar plasmonic resonance caused by retardation effects. ${ }^{38,47}$ Although the samples exhibit a heterogeneous distribution of sizes, the dependence of these parameters on mean NP diameter indicates that the dominant plasmonic resonance is associated with single NP behavior.

For the damping term, a fit of the line width yields $\hbar \gamma(d)=0.18$ $+65 / d \mathrm{eV}$ for $d$ in $\mathrm{nm}$. A similar behavior was seen in small gold and gallium NPs, ${ }^{20,48}$ for which the functional relationship from the summed Drude-Sommerfeld free electron term in eq 3 follows the $d^{-1}$ dependence of the NP surface-to-volume ratio through the damping rate

$$
\hbar \gamma(d)=\hbar \gamma_{0}+2 \frac{A \hbar \nu_{F}}{d}
$$

where $\gamma_{0}$ is the bulk damping rate, and $v_{\mathrm{F}}$ is the Fermi velocity of the electrons. ${ }^{20,48}$ For Ga, the bulk damping constant $\hbar \gamma_{0}=1.54$ $\mathrm{eV}$ is much larger than the fitted value of $0.18 \mathrm{eV}$, indicating significantly reduced damping from the effective layer of $\mathrm{Ga}$ and air. The Ga Fermi velocity ranges between 1.35 and $1.6 \mathrm{~nm} / \mathrm{fs}$, ${ }^{49}$ so assuming an intermediate value $v_{\mathrm{F}}=1.5 \mathrm{~nm} / \mathrm{fs}$ we may estimate the dimensionless size parameter $A=33$. The physical meaning of the $A$ parameter becomes clear from a classical perspective for which $\gamma=\gamma_{\text {bulk }}+v_{f} / L_{\text {eff }}$ where $L_{\text {eff }}$ is an effective scattering length. ${ }^{13,50-53}$ If $L_{\text {eff }}>d / 2$, then the scattering length responsible for the damping is the radius of the NP itself, which is evidently the case here. However, the scattering at the surface may enhance the damping through the effects of adsorbates, oxides, and substrates, shortening the effective scattering length by $A=d / 2 L_{\text {eff }}{ }^{48,52,53}$ The large value of $A$ therefore indicates significant surface damping, caused in part by the hemispherical shape, the monolayer oxide coating, and the strong interaction with the substrate.

\section{ANALYSIS OF VAMM DATA}

A valuable application of the extracted dielectric functions is the prediction of key nonzero and nonunity terms in the Mueller scattering matrix that characterizes how the NPs alter the polarization of the scattered light as a function of wavelength. ${ }^{36,54}$ For incident and scattered fields described by the four component Stokes vectors $S_{i}$ and $S_{s}$, respectively, three terms of the $4 \times 4$ Mueller matrix $\mathrm{M}$ (i.e., $S_{s}=M S_{i}$ ) characterize the degree to which the NPs produce linear horizontal or vertical polarization $\left(m_{12}\right)$ or retard the phase of one polarization relative to the other $\left(m_{33}, m_{34}\right)$. If there is no net depolarization caused by the NPs (i.e., $m_{22}=1$ ), these three terms sufficiently capture the energy-, angle-, and polarization-dependent scattering behavior of the sample.

Figures 3 and 4 show the four Mueller matrix elements mentioned above, obtained through VAMM measurements on

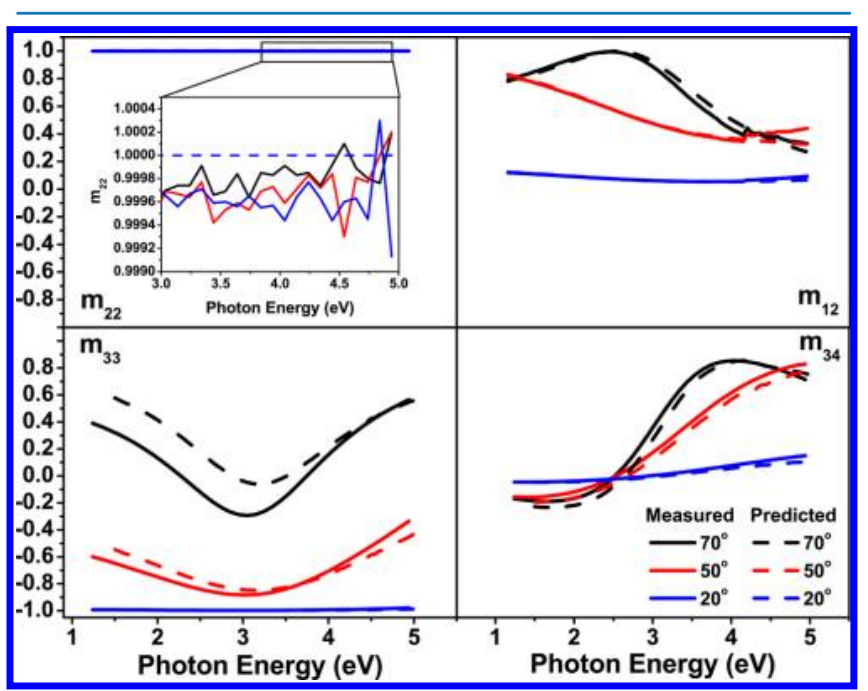

Figure 3. Measured Mueller matrix terms, plotted as a function of incident photon energy for three different incidence/detection angles $\theta$, for sample S2. Overlaid on the data is the prediction of energy- and angle-dependence of each Mueller matrix term using the Lorentz oscillator model.

samples S2 and S3, respectively (solid lines). The dependence of these four terms on energy and incidence/detection angle is plotted in Figure 3 for sample S2, whose behavior was representative of the samples with a unimodal size distribution. For sample $S 2, m_{22}=1$ to within experimental uncertainty $(0.1 \%)$, so the sample causes no depolarizing effects. Considering next the $m_{12}$ spectra, it is not surprising to observe that at near normal incidence $\left(\theta_{i}=20^{\circ}\right)$ the sample does not affect the polarization $\left(m_{12} \approx 0\right)$, but at steeper angles the sample increasingly scatters horizontally polarized light, especially in the region of positive real dielectric function. This is nothing more than scattering near Brewster's angle, which is near $\theta_{i}=70^{\circ}$ for 


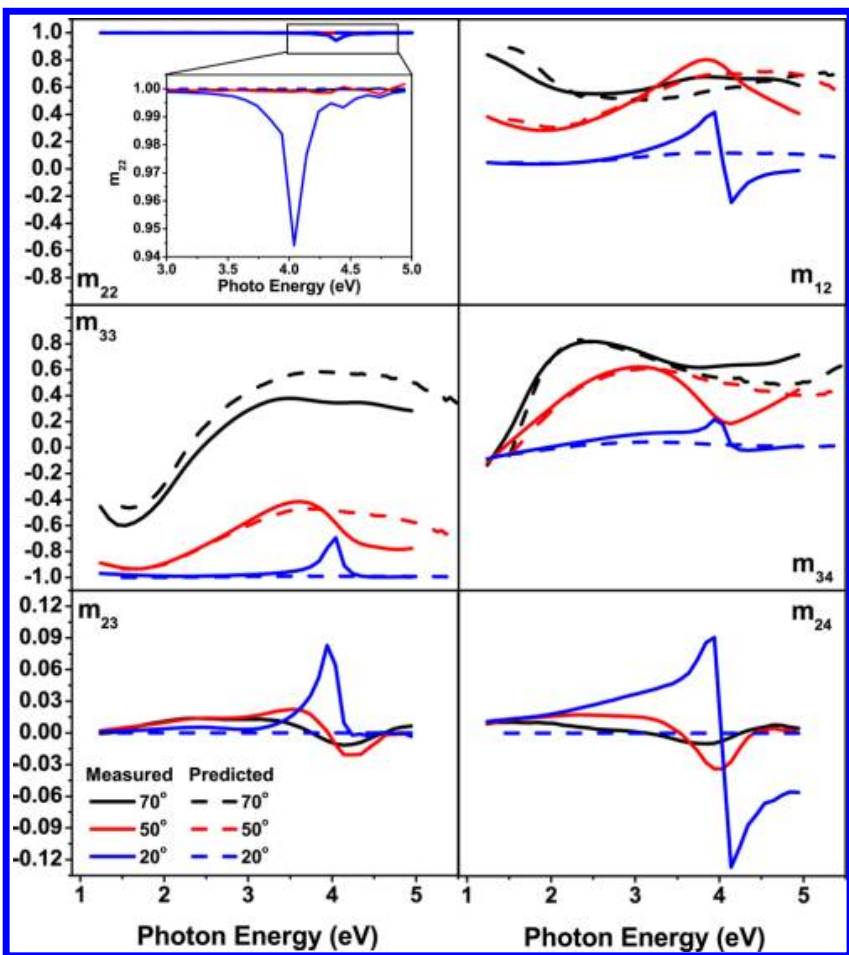

Figure 4. Measured Mueller matrix terms, plotted as a function of incident photon energy for three different incidence/detection angles $\theta_{i}$, for sample S3. Overlaid on the data is the prediction of energy- and angle-dependence of each Mueller matrix term using the Lorentz oscillator model. Note the inclusion of two additional nonzero offdiagonal elements that experimentally exhibited these relationships: $m_{23}$ $\approx-m_{32} \approx-m_{14} \approx m_{41}, m_{24} \approx-m_{42} \approx-m_{13} \approx m_{31}$.

the deduced real dielectric function between 1.5 and $3.0 \mathrm{eV}$. The reduced polarizing behavior at higher energies is a manifestation of the broad absorption by the LSPR. Nevertheless, the sample is more polarizing than a film of Ga because the high angle of incidence excites a vertically oriented out-of-plane dipolar mode that preferentially absorbs p-polarized light. Next consider $m_{33}$ and $m_{34}$. At near normal incidence $\left(\theta_{i}=20^{\circ}\right), m_{33} \approx-1$ and $m_{34}$ $\approx 0$ for all wavelengths. This is the classic behavior of a half-wave retarder produced by the well-known half-wave phase shift at this interface. At steeper angles, the degree of phase retardation evolves in an energy-dependent manner. The valley $m_{33}$ and the peak $m_{34}$ for the steepest incident angle $\left(\theta_{i}=70^{\circ}\right)$ corresponds to the peak in the dielectric function related to the plasmon resonance near $3.5 \mathrm{eV}$.

Overlaid on the data (dashed lines) are the estimates of these terms derived from the dielectric functions in Figure 2(c) obtained through the Lorentz oscillator model described above. For sample S2 with a unimodal size distribution, the energy- and angle-dependent behavior of these four terms is very well reproduced by this analysis, suggesting almost all the observed scattering behavior may be understood as a response of free electrons within noninteracting NPs to the incident field in a manner affected by NP geometry, the substrate, and surface adsorbates. This behavior is reproduced in all the samples with unimodal size distributions; however, spectrally isolated deviations from this behavior were observed in the samples with bimodal size distributions.

\section{DEPOLARIZING BEHAVIOR OF NANOPARTICLES WITH A BIMODAL SIZE DISTRIBUTION}

To understand these deviations for bimodal sample S3, consider first that the Lorentz oscillator model with $\theta_{i}=70^{\circ}$ reproduces the energy dependence of the traditional ellipsometric parameters $\Psi$ and $\Delta$ quite well (Figure 2(a)). It even reproduces the measured $\theta_{i}=70^{\circ}$ VAMM data (Figure 4), but the agreement between measured data and predicted Lorentzian behavior deteriorates as the angle of incidence is reduced. Figure 4 shows that the deviation from unity in $m_{22}$ occurs over a remarkably narrow frequency range near $4.0 \mathrm{eV}$ and only for near normal incidence, both strong indicators that depolarizing behavior is occurring that cannot be explained by the Lorentz oscillator model. Common causes of depolarization include surface roughness, film thickness inhomogeneity, backside reflection from a weakly absorbing substrate, and plasmon resonance of nanostructures. ${ }^{17,25}$ Note that the feature cannot be explained by enhanced coupling to the out-of-plane mode; otherwise, the feature would have grown stronger as $\theta_{i}$ increased. Instead, the feature vanishes at larger $\theta_{i}$ due to the shadowing effects of the larger NPs and the increasing contribution of their out-of-plane LSPR mode whose maximum occurs at $4.75 \mathrm{eV}$.

To explore the cause of this feature, we measured the percentage of light scattered into all angles as a function of energy for an incidence angle of $\theta_{i}=40^{\circ}$ to ascertain the specular and nonspecular scattering strength of this sample (Figure 5). Notice

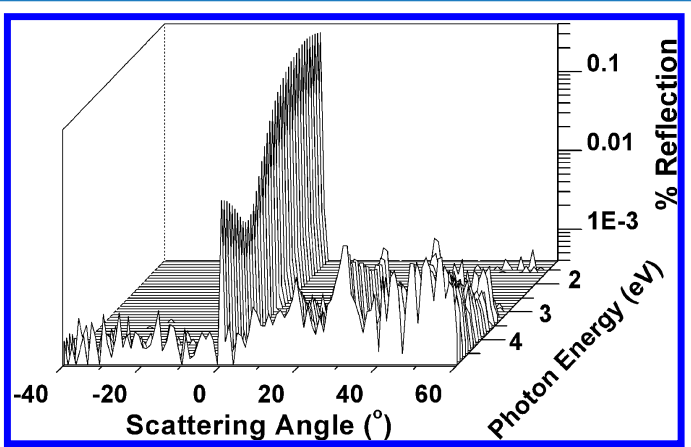

Figure 5. Measurement of the specular $\left(\chi=0^{\circ}\right)$ and nonspecular reflection percentage, plotted logarithmically as a function of energy from sample $\mathrm{S} 3$ for $\theta_{i}=40^{\circ}$. The data is taken with $0.1 \mathrm{eV}$ energy and $1^{\circ}$ angular resolution.

that for low energies the vast majority of light is scattered in the specular direction $\left(\theta_{\mathrm{i}}-\theta_{\text {reflection }}=\chi=0^{\circ}\right.$ in this plot $)$. However, for higher energies the proportion of light scattered in the specular direction drops precipitously. This drop may be largely attributed to both increased absorption by the LSPR and increased sensitivity to surface roughness at shorter wavelengths. However, notice that scattering in nonspecular directions simultaneously increases, something that is not seen in unimodal samples or the bimodal samples at larger $\theta_{i}$. Scattering at angles greater than $\chi=50^{\circ}$ represents light coupled into the substrate, while light scattered narrowly around $\chi=20^{\circ}$ and especially $\chi=$ $30^{\circ}\left(\theta_{\text {reflection }}=60^{\circ}\right.$ and $\theta_{\text {reflection }}=70^{\circ}$, respectively $)$ propagates into free space. Nonspecular scattering is not seen at any angle between specular $\left(\chi=0^{\circ}\right)$ and sample normal $\left(\chi=-40^{\circ}\right)$.

The fact that none of this behavior is seen in samples with unimodal size distributions suggests some new effect is active in the bimodal samples for near normal illumination. In fact, this depolarizing $\left(m_{22}<1\right)$, nonspecular scattering behavior for small $\theta_{i}$ is reproducibly seen in all bimodal samples and evidently arises 
from interactions between large NPs and the surrounding halo of smaller NPs. The feature red-shifts with increasing average NP size (Figure 6), confirming that it is associated with the

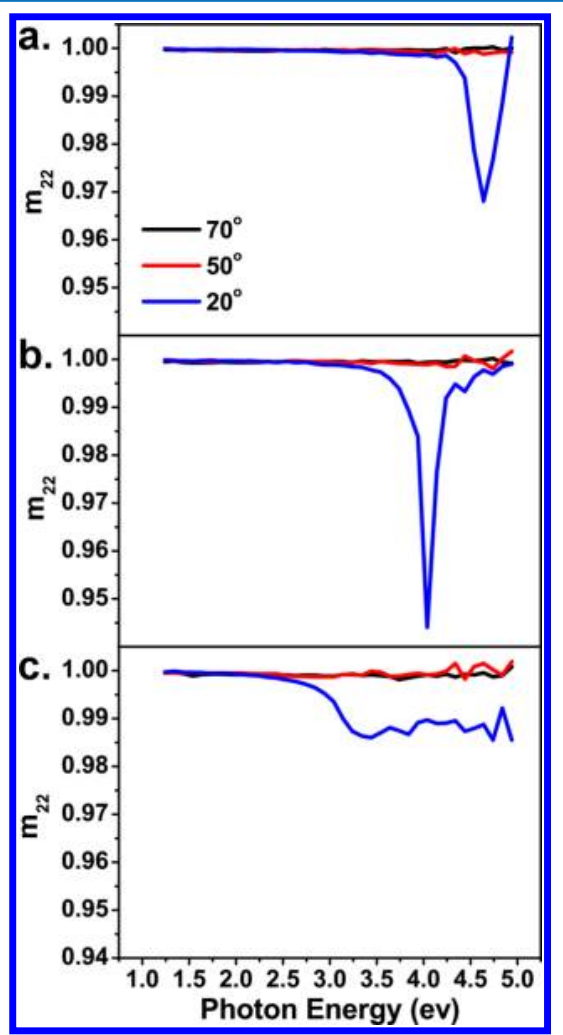

Figure 6. Measured $\theta_{i}=20^{\circ}, 50^{\circ}$, and $70^{\circ} m_{22}$ depolarization feature for three samples with bimodal size distributions of increasing average diameter $d=90(\mathrm{a}), 140(\mathrm{~S} 3, \mathrm{~b})$, and $228 \mathrm{~nm}(\mathrm{c})$.

plasmonic response and the increased scattering contribution of the NPs, both of which redshift and increase as the size of the NPs increases. As additional evidence of this, consider that the depolarizing feature in S3 disappears for large $\theta_{i}$ where the larger NPs shadow the smaller NPs. Moreover, the in-plane dipoles induced by the parallel component of the incident light are responsible for interparticle coupling, and close to grazing $\theta_{i}$ these dipoles and interparticle couplings are weakest. By contrast, for $\theta_{i}$ near normal incidence the shadowing is removed, the inplane dipoles are strongly excited, the coupling is strongest, and the depolarizing feature appears. Besides this, the NPs in the unimodal samples are small and only weakly scatter light in the analyzed spectral range, while the larger NPs in the bimodal samples scatter more effectively, leading to multiple scattering and interparticle coupling.

Mueller matrix polar decomposition helps clarify and quantify these behaviors by factoring the complex optical response into a combination of three components: a depolarizer, a retarder and a diattenuator. ${ }^{35}$ Our measurements indicate that bimodal sample S3 exhibits significant depolarization, retardance, and diattenuation effects which cannot be characterized solely by spectroscopic ellipsometry. In particular, the depolarization power $\Delta_{\mathrm{D}}$ derived from the trace of the depolarization Mueller submatrix $M_{\Delta}$ quantifies the degree of depolarization. At $4.04 \mathrm{eV}$ and $\theta_{i}=$ $20^{\circ}$, nondepolarizing sample S2 exhibits little depolarization $\left(\Delta_{D}\right.$ $=0.0004)$ while sample S3 exhibits fairly strong depolarization $\left(\Delta_{\mathrm{D}}=0.19\right)$. The latter coincides with the largest nonunity values of $m_{22}(=0.94)$ and largest nonzero values of the off-diagonal terms in the Mueller matrix (Figure 4). Recent research has also shown strong retardance for nanostructures with separate plasmon modes simultaneously excited. ${ }^{55}$

So why does the behavior occur over such a narrow energy range? It has already been shown that the strongest local field enhancements occur in the space between asymmetric NP dimers in which the larger NP acts as the "antenna" and the smaller NP acts as the "resonator." ${ }^{56}$ It has also been shown that narrow resonances occur when NPs are tightly coupled, resonances that grow stronger as the central NP grows larger than the NPs in the surrounding halo. ${ }^{8,57}$ For these reasons, strong coupling among asymmetric NP clusters may explain the observed narrow frequency depolarizing behavior that redshifts as the size of the associated NPs grows (Figure 6). Similarly narrow resonances were observed in the far field depolarization produced by arrays of symmetric gold dimers and were likewise ascribed to the efficient excitation of associated LSPR modes. ${ }^{58}$ In that work, the percentage of depolarization induced by the dimers $\Delta_{\mathrm{dp}}=100 \%\left[1-\left(\alpha^{2}+\beta^{2}+\gamma^{2}\right)^{1 / 2}\right]$ was shown to be largest when the excitation of the dimer LSPR was largest $(\alpha, \beta, \gamma$ depend only on $\Psi$ and $\Delta) .{ }^{59}$ We find that $\Delta_{d p} \ll 1 \%$ for both unimodal samples $S 1, S 2$ and at most energies and incidence angles for S3; however, the largest depolarization for S3 $\left(\Delta_{\mathrm{dp}}=\right.$ $29.5 \%$ ) occurs precisely where $m_{22}$ deviates the most from unity: at $4.04 \mathrm{eV}$ and $\theta_{i}=20^{\circ}$.

Evidently the depolarizing feature is associated with particularly strong local field enhancements. Further confirmation of this comes from recently reported measurements of UV surface-enhanced Raman spectra of the analyte crystal violet deposited on all three samples. ${ }^{31}$ In that study, the strongest Raman enhancement occurred for a laser energy $(3.815 \mathrm{eV}=325$ $\mathrm{nm}$ ) that nearly coincides with the energy of the depolarizing feature in the bimodal sample S3. This suggests that NP ensembles containing strongly coupled asymmetric NP dimers or $n$-mers produce strong local field enhancements when their depolarizing behavior coincides with the laser energy and $\theta_{i}$ is small. Our measurements show how these depolarizing features may be observed through VASE/VAMM techniques.

\section{CONCLUSIONS}

We have reported a series of UV/vis VASE and VAMM measurements on self-assembled irregular ensembles of truncated spherical gallium NPs grown by MBE on sapphire substrates. These samples, whose constituent unimodal or bimodal distribution of NP sizes was controlled by deposition time, exhibited tunable localized surface plasmon resonances. Using a Lorentz oscillator model to parametrize the dielectric response of the ensemble, relationships were found between NP size and the deduced Lorentzian parameters (resonant frequency, damping, oscillator strength) for most unimodal and bimodal samples at most frequencies and angles of incidence. The Lorentz model also accurately predicts the frequency- and angle-dependence of the critical terms in the measured Mueller matrix characterizing the polarimetric scattering by these samples. These findings indicate that the plasmonic behavior of self-assembled gallium NP ensembles with a unimodal size distribution $<100 \mathrm{~nm}$ in diameter is dominated by the isolated, single-particle response of the numerous NPs of average diameter.

However, for samples exhibiting a bimodal distribution of larger NPs surrounded by a halo of smaller NPs, deviations from this Lorentzian behavior are observed, but only at certain combinations of incidence angle and frequency. Nonspecular 
scattering and depolarizing features observed in the Mueller matrix spectra for these same conditions suggest that electromagnetic coupling between NPs alters the plasmonic performance. ${ }^{56}$ Considering that these behaviors occur precisely where UV surface-enhanced Raman spectra indicated strong local field enhancements, ${ }^{31}$ we conclude that VAMM analyses may be used to characterize heterogeneous arrays of metal NPs to ascertain the spectral regions and optical geometries that produce the strongest local field enhancements.

\section{AUTHOR INFORMATION}

\section{Corresponding Author}

*E-mail: everitt@phy.duke.edu.

\section{Notes}

The authors declare no competing financial interest.

\section{ACKNOWLEDGMENTS}

The authors would like to thank M. Bloemer for facilitating the use of the VASE instrument. F.M. and J.M.S. acknowledge funding from USAITCA (project no. W911NF-13-1-0245). This work was partially supported by the Army's competitive in-house innovative laboratory research program and the Katherine Goodman Stern fellowship program.

\section{REFERENCES}

(1) Moskovits, M. Surface-Enhanced Spectroscopy. Rev. Mod. Phys. 1985, 57, 783-826.

(2) Kneipp, K.; Wang, Y.; Kneipp, H.; Perelman, L.; Itzkan, I.; Dasari, R.; Feld, M. Single Molecule Detection Using Surface-Enhanced Raman Scattering (SERS). Phys. Rev. Lett. 1997, 78, 1667-1670.

(3) Stiles, P. L.; Dieringer, J. A.; Shah, N. C.; van Duyne, R. P. SurfaceEnhanced Raman Spectroscopy. Annu. Rev. Anal. Chem. 2008, 1, 601626.

(4) Lal, S.; Grady, N. K.; Kundu, J.; Levin, C. S.; Lassiter, J. B.; Halas, N. J. Tailoring Plasmonic Substrates for Surface Enhanced Spectroscopies. Chem. Soc. Rev. 2008, 37, 898-911.

(5) Linic, S.; Christopher, P.; Ingram, D. B. Plasmonic-Metal Nanostructures for Efficient Conversion of Solar to Chemical Energy. Nat. Mater. 2011, 10, 911-921.

(6) Fort, E.; Grésillon, S. Surface Enhanced Fluorescence. J. Phys. D: Appl. Phys. 2007, 41, 013001.

(7) Ozbay, E. Plasmonics: Merging Photonics and Electronics at Nanoscale Dimensions. Science 2006, 311, 189-193.

(8) Lassiter, J. B.; Sobhani, H.; Knight, M. W.; Mielczarek, W. S.; Nordlander, P.; Halas, N. J. Designing and Deconstructing the Fano Lineshape in Plasmonic Nanoclusters. Nano Lett. 2012, 12, 1058-1062.

(9) Moreau, A.; Ciracì, C.; Mock, J. J.; Hill, R. T.; Wang, Q.; Wiley, B. J.; Chilkoti, A.; Smith, D. R. Controlled-Reflectance Surfaces with FilmCoupled Colloidal Nanoantennas. Nature 2012, 492, 86-89.

(10) Pelton, M.; Aizpurua, J.; Bryant, G. Metal Nanoparticle Plasmonics. Laser Photonics Rev. 2008, 2, 136-159.

(11) Nelayah, J.; Kociak, M.; Stéphan, O.; García de Abajo, F.J.; Tencé, M.; Henrard, L.; Taverna, D.; Pastoriza-Santos, I.; Liz-Marzán, L. M.; Colliex, C. Mapping Surface Plasmons on a Single Metallic Nanoparticle. Nat. Phys. 2007, 3, 348-353.

(12) Yamamoto, N.; Araya, K.; García de Abajo, F. Photon Emission from Silver Particles Induced by a High-Energy Electron Beam. Phys. Rev. B 2001, 64, 205419.

(13) Zeman, E. J.; Schatz, G. C. An Accurate Electromagnetic Theory Study of Surface Enhancement Factors for Silver, Gold, Copper, Lithium, Sodium, Aluminum, Gallium, Indium, Zinc, and Cadmium. J. Phys. Chem. 1987, 91, 634-643.

(14) Schatz, G. C.; Young, M. A.; van Duyne, R. P. Electromagnetic Mechanism of SERS. Top. Appl. Phys. 2006, 103, 19-45.
(15) Huang, S. Y.; Wu, B.-I.; Zhang, B.; Lee, Y. H.; Liberman, V.; Rothschild, M. An Analytical Method to Study the Effects of a Substrate in Surface-Enhanced Raman Scattering. J. Appl. Phys. 2009, 106, 114306.

(16) Sanz, J. M.; Albella, P.; Moreno, F.; Saiz, J. M.; González, F. Application of the Polar Decomposition to Light Scattering Particle Systems. J. Quant. Spectrosc. Radiat. Transfer 2009, 110, 1369-1374.

(17) Lal, S.; Link, S.; Halas, N. J. Nano-Optics from Sensing to Waveguiding. Nat. Photonics 2007, 1, 641-648.

(18) Willets, K. A.; van Duyne, R. P. Localized Surface Plasmon Resonance Spectroscopy and Sensing. Annu. Rev. Phys. Chem. 2007, 58, 267-297.

(19) Albella, P.; Moreno, F.; Saiz, J.; González, F. Surface Inspection by Monitoring Spectral Shifts of Localized Plasmon Resonances. Opt. Express 2008, 16, 12872-12879.

(20) Tonova, D.; Patrini, M.; Tognini, P.; Stella, A.; Cheyssac, P.; Kofman, R. Ellipsometric Study of Optical Properties of Liquid Ga Nanoparticles. J. Phys.: Condens. Mater. 1999, 11, 2211-2222.

(21) Azzam, R. M. A.; Bashara, N. M. Ellipsometry and Polarized Light; Elsevier: Amsterdam, 1987.

(22) Fujiwara, H. Spectroscopic Ellipsometry: Principles and Applications; John Wiley \& Sons: West Sussex, England, 2007.

(23) Kürbitz, S.; Porstendorfer, J.; Berg, K. J.; Berg, G. Determination of Size and Concentration of Copper Nanoparticles Dispersed in Glasses Using Spectroscopic Ellipsometry. Appl. Phys. B: Laser Opt. 2001, 73, 333-337.

(24) Oates, T. W. H.; Wormeester, H.; Arwin, H. Characterization of Plasmonic Effects in Thin Films and Metamaterials Using Spectroscopic Ellipsometry. Prog. Surf. Sci. 2011, 86, 328-376.

(25) Oates, T. W. H.; Mücklich, A. Evolution of Plasmon Resonances during Plasma Deposition of Silver Nanoparticles. Nanotechnology 2005, $16,2606-2611$

(26) Oates, T. W. H.; Ryves, L.; Bilek, M. M. M. Dielectric Functions of a Growing Silver Film Determined Using Dynamic in Situ Spectroscopic Ellipsometry. Opt. Express 2008, 16, 2302-2314.

(27) Choi, S.; Kim, T.-H.; Brown, A. S.; Everitt, H. O.; Losurdo, M.; Bruno, G.; Moto, A. Kinetics of Gallium Adsorption and Desorption on (0001) Gallium Nitride Surfaces. Appl. Phys. Lett. 2006, 89, 181915181915.

(28) Choi, S.; Kim, T.-H.; Everitt, H. O.; Brown, A. S.; Losurdo, M.; Brun, G.; Moto, A. Kinetics of Gallium Adlayer Adsorption/Desorption on Polar and Nonpolar GaN Surfaces. J. Vac. Sci. Technol. B 2007, 25, 969-973.

(29) Wu, P. C.; Kim, T.-H.; Brown, A. S.; Losurdo, M.; Bruno, G.; Everitt, H. O. Real-Time Plasmon Resonance Tuning of Liquid Ga Nanoparticles by in Situ Spectroscopic Ellipsometry. Appl. Phys. Lett. 2007, 90, 103119.

(30) Wu, P. C.; Losurdo, M.; Kim, T.-H.; Choi, O.; Bruno, G.; Brown, A. S. In Situ Spectroscopic Ellipsometry to Monitor Surface Plasmon Resonant Group-III Metals Deposited by Molecular Beam Epitaxy. J. Vac. Sci. Technol. B 2007, 25, 1019-1023.

(31) Yang, Y.; Callahan, J. M.; Kim, T.-H.; Brown, A. S.; Everitt, H. O. Ultraviolet Nanoplasmonics: a Demonstration of Surface-Enhanced Raman Spectroscopy, Fluorescence, and Photodegradation Using Gallium Nanoparticles. Nano Lett. 2013, 13, 2837-2841.

(32) Wu, P. C.; Khoury, C. G.; Kim, T.-H.; Yang, Y.; Losurdo, M.; Bianco, G. V.; Vo-Dinh, T.; Brown, A. S.; Everitt, H. O. Demonstration of Surface-Enhanced Raman Scattering by Tunable, Plasmonic Gallium Nanoparticles. J. Am. Chem. Soc. 2009, 131, 12032-12033.

(33) Losurdo, M.; Bergmair, M.; Bruno, G.; Cattelan, D.; Cobet, C.; Martino, A.; Fleischer, K.; Dohcevic-Mitrovic, Z.; Esser, N.; Galliet, M.; Gajic, R.; Hemzal, D.; Hingerl, K.; Humlicek, J.; Ossikovski, R.; Popovic, Z. V.; Saxl, O. Spectroscopic Ellipsometry and Polarimetry for Materials and Systems Analysis at the Nanometer Scale: State-of-the-Art, Potential, and Perspectives. J. Nanopart. Res. 2009, 11, 1521-1554.

(34) Oates, T. W. H.; Ranjan, M.; Facsko, S.; Arwin, H. Highly Anisotropic Effective Dielectric Functions of Silver Nanoparticle Arrays. Opt. Express 2011, 19, 2014-2028.

(35) Lu, S.-Y.; Chipman, R. A. Interpretation of Mueller Matrices Based on Polar Decomposition. J. Opt. Soc. Am. A 1996, 13, 1106-1113. 
(36) Laskarakis, A.; Logothetidis, S.; Pavlopoulou, E.; Gioti, M. Mueller Matrix Spectroscopic Ellipsometry: Formulation and Application. Thin Solid Films 2004, 455, 43-49.

(37) Little, S. A.; Collins, R. W.; Marsillac, S. Analysis of Interband, Intraband, and Plasmon Polariton Transitions in Silver Nanoparticle Films via in Situ Real-Time Spectroscopic Ellipsometry. Appl. Phys. Lett. 2011, 98, 101910 .

(38) Albella, P.; Garcia-Cueto, B.; González, F.; Moreno, F.; Wu, P. C.; Kim, T.-H.; Brown, A. S.; Yang, Y.; Everitt, H. O.; Videen, G. Shape Matters: Plasmonic Nanoparticle Shape Enhances Interaction with Dielectric Substrate. Nano Lett. 2011, 11, 3531-3537.

(39) Knight, M. W.; Liu, L.; Wang, Y.; Brown, L.; Mukherjee, S.; King, N. S.; Everitt, H. O.; Nordlander, P.; Halas, N. J. Aluminum Plasmonic Nanoantennas. Nano Lett. 2012, 12, 6000-6004.

(40) McMahon, J. M.; Schatz, G. C.; Gray, S. K. Plasmonics in the Ultraviolet with the Poor Metals $\mathrm{Al}, \mathrm{Ga}$, in, $\mathrm{Sn}, \mathrm{Tl}, \mathrm{Pb}$, and Bi. Phys. Chem. Chem. Phys. 2013, 15, 5415-5423.

(41) Sanz, J. M.; Ortiz, D.; de la Osa, R. A.; Saiz, J. M.; González, F.; Brown, A. S.; Losurdo, M.; Everitt, H. O.; Moreno, F. UV Plasmonic Behavior of Various Metal Nanoparticles in the Near-and Far-Field Regimes: Geometry and Substrate Effects. J. Phys. Chem. C 2013, 117, 19606-19615.

(42) Wu, P. C.; Losurdo, M.; Kim, T.-H.; Giangregorio, M.; Bruno, G.; Everitt, H. O.; Brown, A. S. Plasmonic Gallium Nanoparticles on Polar Semiconductors: Interplay Between Nanoparticle Wetting, Localized Surface Plasmon Dynamics, and Interface Charge. Langmuir 2009, 25, 924-930.

(43) Parravicini, G. B.; Stella, A.; Ghigna, P.; Spinolo, G.; Migliori, A.; D'Acapito, F.; Kofman, R. Extreme Undercooling (Down to $90 \mathrm{~K}$ ) of Liquid Metal Nanoparticles. Appl. Phys. Lett. 2006, 89, 033123.

(44) Ratke, L.; Voorhees, P. W. Growth and Coarsening: Ripening in Material Processing; Springer Verlag: Berlin, 2002.

(45) Ellipsometry at the Nanoscale; Losurdo, M.; Hingerl, K., Eds.; Springer Verlag: Berlin, 2013.

(46) Oates, T. W. H.; Sugirne, H.; Noda, S. Combinatorial SurfaceEnhanced Raman Spectroscopy and Spectroscopic Ellipsometry of Silver Island Films. J. Phys. Chem. C 2009, 113, 4820-4828.

(47) Bohren, C. F.; Huffman, D. R. Absorption and Scattering of Light by Small Particles; John Wiley \& Sons: New York, 1983.

(48) Berciaud, S.; Cognet, L.; Tamarat, P.; Lounis, B. Observation of Intrinsic Size Effects in the Optical Response of Individual Gold Nanoparticles. Nano Lett. 2005, 5, 515-518.

(49) Burma, N. G.; Avramenko, Y. A.; Petrishin, A. I.; Fil, V. D. Effect of Resonance Electron-Phonon Interactions on the Electronic Spectra of Tungsten and Gallium. Low Temp. Phys. 2012, 38, 437-441.

(50) Kreibig, U. Electronic Properties of Small Silver Particles: Optical Constants and Their Temperature Dependence. J. Phys. F: Met. Phys. 1974, 4, 999-1014.

(51) Kreibig, U.; Vollmer, M. Optical Properties of Metal Clusters; Springer: Berlin, 1995.

(52) Kraus, W. A.; Schatz, G. C. Plasmon Resonance Broadening in Spheroidal Metal Particles. Chem. Phys. Lett. 1983, 99, 353-357.

(53) Kraus, W. A.; Schatz, G. C. Plasmon Resonance Broadening in Small Metal Particles. J. Chem. Phys. 1983, 79, 6130-6139.

(54) Aslan, M. M.; Mengüç, M. P.; Videen, G. Characterization of Metallic Nano-Particles via Surface Wave Scattering: B. Physical Concept and Numerical Experiments. J. Quant. Spectrosc. Radiat. Transfer 2005, 93, 207-217.

(55) Soni, J.; Ghosh, S.; Mansha, S.; Kumar, A.; Dutta Gupta, S.; Banerjee, A.; Ghosh, N. Enhancing Spin-Orbit Interaction of Light by Plasmonic Nanostructures. Opt. Lett. 2013, 38, 1748-1750.

(56) Sun, G.; Khurgin, J. B. Optimization of the Nanolens Consisting of Coupled Metal Nanoparticles: an Analytical Approach. Appl. Phys. Lett. 2011, 98, 153115.

(57) Ye, J.; Wen, F.; Sobhani, H.; Lassiter, J. B.; Dorpe, P. V.; Nordlander, P.; Halas, N. J. Plasmonic Nanoclusters: Near Field Properties of the Fano Resonance Interrogated with SERS. Nano Lett. 2012, 12, 1660-1667.
(58) Walsh, G. F.; Forestiere, C.; Dal Negro, L. Plasmon-Enhanced Depolarization of Reflected Light From Arrays of Nanoparticle Dimers. Opt. Express 2011, 19, 21081-21090.

(59) Joerger, R.; Forcht, K.; Gombert, A.; Köhl, M.; Graf, W. Influence of Incoherent Superposition of Light on Ellipsometric Coefficients. Appl. Opt. 1997, 36, 319-327. 